\title{
Mini Open Foraminotomy for Cervical Radiculopathy: A Comparison of Large Tubular and TrimLine Retractors
}

\author{
Masashi Uehara ${ }^{1}$, Jun Takahashi ${ }^{1}$, Shugo Kuraishi ${ }^{1}$, Masayuki Shimizu ${ }^{1}$, Shota Ikegami ${ }^{1}$, \\ Toshimasa Futatsugi ${ }^{1}$, Kaoru Aoki ${ }^{1}$, Keijiro Mukaiyama ${ }^{1}$, Nobuhide Ogihara ${ }^{2}$, \\ Hiroyuki Hashidate ${ }^{3}$, Hiroki Hirabayashi ${ }^{4}$, Hiroyuki Kato ${ }^{1}$ \\ ${ }^{1}$ Department of Orthopaedic Surgery, Shinshu University School of Medicine, Matsumoto, Japan \\ ${ }^{2}$ Department of Orthopaedic Surgery, Ina Central Hospital, Ina, Japan \\ ${ }^{3}$ Department of Orthopaedic Surgery, Shinonoi General Hospital, Nagano, Japan \\ ${ }^{4}$ Department of Orthopaedic Surgery, Marunouchi Hospital, Matsumoto, Japan
}

\section{Study Design: Retrospective chart review.}

Purpose: A comparison of mini open foraminotomy (MOF) for cervical radiculopathy using either large tubular (LT) or TrimLine (TL) retractors.

Overview of Literature: Posterior foraminotomy relieves compression of the cervical nerve root in radiculopathy patients. However, invasion of the paravertebral muscle may cause major problems in these patients. To address these problems, we performed MOF.

Methods: Twenty cervical radiculopathy patients (16 male and 4 female) who underwent MOF between May 2004 and August 2011 were assigned to LT and TL groups. Each group contained 10 subjects. Surgical and clinical outcomes were compared.

Results: The average operating time in the TL group was significantly shorter than that in the LT group. The final follow-up mean neck disability indices significantly improved compared to the preoperative values (LT group, $12.0 \pm 7.8$ vs. $28.0 \pm 9.4$; TL group, $6.0 \pm 5.9$ vs. 21.9 \pm 10 ). The final follow-up neck pain visual analog scale (VAS) scores also decreased significantly from the preoperative of $8.0 \pm 1.5$ and $2.5 \pm 2.5$ to the final follow-up values of $2.2 \pm 2.2$ and $1.0 \pm 2.5$ in the $L T$ and TL groups, respectively. The recovery rate for the neck pain VAS score was $70.0 \pm 31.9$ in the LT group and $87.0 \pm 32.0$ in the TL group, thus suggesting no significant difference between the two groups.

Conclusions: MOF with the TL retractor is an easy and safe procedure. Furthermore, the use of the TL retractor allows for a minimally invasive and effective surgical treatment of cervical radiculopathy patients.

Keywords: Cervical radiculopathy; Postoperative neck pain; Paravertebral muscle; Mini open framinotomy; Less invasive

\section{Introduction}

Posterior foraminotomy through partial resection of the medial aspect of the facet joint was described by Fryk- holm [1] and Scoville [2] as a method aimed at relieving compression of the cervical nerve root in radiculopathy patients. Despite the usefulness of this procedure, the related morbidity resulting from iatrogenic soft tissue

Received Oct 26, 2014; Revised Nov 28, 2014; Accepted Dec 10, 2014

Corresponding author: Jun Takahashi

Department of Orthopaedic Surgery, Shinshu University School of Medicine,

3-1-1 Asahi, Matsumoto, 390-8621, Japan

Tel: +81-263-37-2659, Fax: +81-263-35-8844, E-mail: jtaka@shinshu-u.ac.jp 
injury, including the paraspinal muscle injury caused by its extensive detachment from the posterior aspect of the cervical spine, has become a major problem [3-5]. Therefore, minimally invasive spinal procedures are being developed to effectively treat cervical spine disease. These developments have led to surgical approaches that result in less tissue trauma, reduce postoperative pain and discomfort, shorten hospital stays, and allow a quicker return to normal activities. However, the learning curve is steep for the surgeon conducting such operations. Therefore, we developed mini open foraminotomy (MOF), a procedure that can be more easily performed under direct vision and through smaller incisions. This minimally invasive technique was proven to be effective for the treatment of cervical radiculopathy [6]. In this retrospective study, we present a comparison of large tubular (LT) and TrimLine (TL) retractors for the use in MOF performed for cervical radiculopathy.

\section{Materials and Methods}

This study was approved by Shinshu university hospital Investigational Review Board. Included were twentyfour consecutive patients (20 male and 4 female; mean age, $51.7 \pm 10.4$ years; range, $30-78$ years) with foraminal stenosis confirmed by imaging and neurological findings and radicular pain that had remained refractory after more than 3 months of conservative therapy.

Inclusion criteria were cervical disc herniation or cervical spondylotic radiculopathy at 1 intervertebral level. Traffic accident cases as well as those with more than 2 intervertebral level lesions were excluded. Furthermore, 4 cases were excluded because of short follow-up peri- ods ( $<10$ months). The data of the remaining 20 patients (16 male and 4 female; mean age, $53.5 \pm 9.2$ years; range, 39-78 years) who underwent MOF ( 1 intervertebral level) for the treatment of cervical radiculopathy between May 2004 and August 2011 were retrospectively reviewed.

METRx LT retractors (22 mm diameter; Medtronic, Memphis, TN, USA) (Fig. 1A) were used in 10 cases (LT group, 7 male and 3 female; mean age, $52.6 \pm 7.6$ years; range, 39-65 years) from December 2004 to May 2008. TL retractors (Medtronic) that simplify the surgical technique (Fig. 1B) were used in the other 10 patients (TL group, 9 male and 1 female; mean age, $54.3 \pm 10.9$ years; range, 40-78 years) who underwent MOF from May 2004 to August 2011 (starting from June 2008, all cases of MOF were performed with TL retractors). The surgical technique has been described by Takahashi et al. [6].

\section{The use of LT retractors}

A $3 \mathrm{~cm}$ longitudinal skin incision was made about $1.5 \mathrm{~cm}$ laterally to the center. The fascia was then incised by electrocautery; the paravertebral muscles were retracted with a dilater, finger, and/or Cobb elevator; and the METRx LT retractors were inserted.

\section{The use of TL retractors}

A $5 \mathrm{~cm}$ longitudinal skin incision was made about 1.5 $\mathrm{cm}$ laterally to the center. The paravertebral muscles were detached from the spinous processes and lamina and retracted longitudinally with TL retractors.

Neck disability indices (NDI) (full score, 50 points) were assessed and visual analog scale (VAS) scores of neck
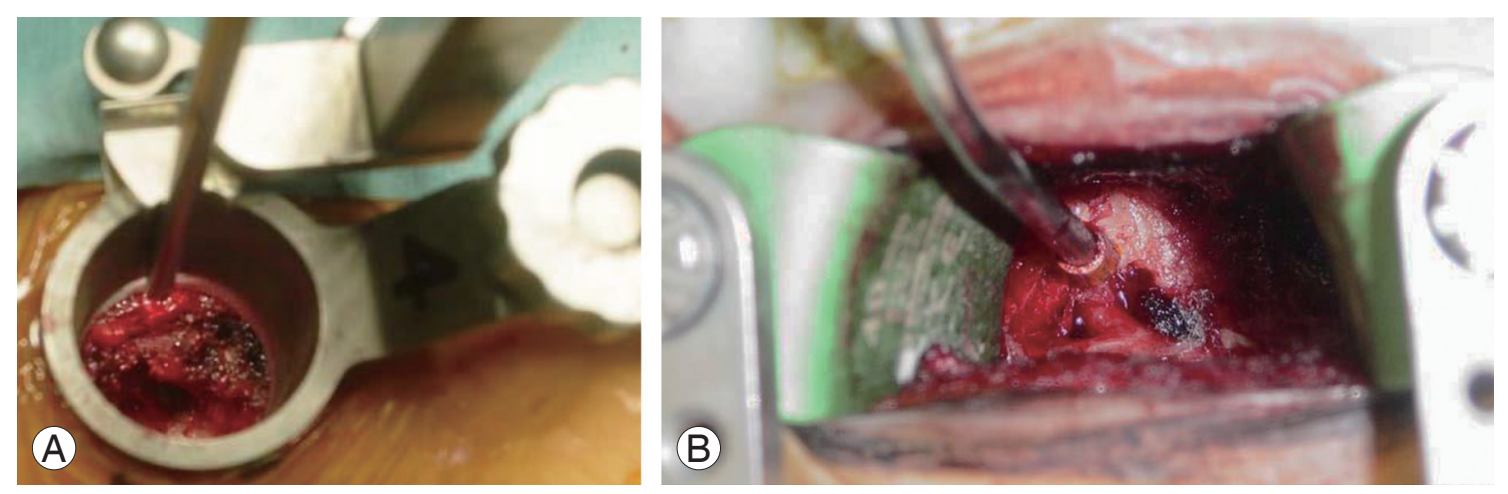

Fig. 1. Intraoperative photographs. (A) Mini open foraminotomy (MOF) was performed with a large tubular retractor. (B) MOF with a TrimLine retractor was performed using a $5 \mathrm{~cm}$ skin incision. 
Table 1. The characteristics of the study population

\begin{tabular}{lccc} 
Variable & LT retractor group $(\mathrm{n}=10)$ & TL retractor group $(\mathrm{n}=10)$ & $p$-value \\
\hline Mean age (yr) & $52.6 \pm 7.6$ & $54.3 \pm 10.9$ & 1.00 \\
\hline Follow-up (mo) & $41.8 \pm 24.7$ & $33.0 \pm 19.3$ & 0.393 \\
\hline Surgical time (min) & $86.2 \pm 30.0$ & $60.5 \pm 22.5$ & 0.034 \\
Intraoperative blood loss (g) & $42.0 \pm 45.4$ & $43.5 \pm 43.8$ & 0.796 \\
\hline Preoperative NDI (score) & $28.0 \pm 9.4$ & $21.9 \pm 10.3$ & 0.164 \\
\hline
\end{tabular}

Values are presented as mean \pm standard deviation.

LT, large tubular; TL, TrimLine; NDI, neck disability index.

a)Determined by the Mann-Whitney U-test.

and arm pain were obtained before the surgery and at the final follow-up. Recovery rates were calculated using a modified Hirabayashi method [7], which compared preand postoperative VAS scores according to the following equation:

Recovery rate $(\%)=[(10-$ postoperative score $)-(10-$ preoperative score $)] \times 100 /[($ full score-(10-preoperative score)]

The operating time, intraoperative blood loss, length of hospital stay, and C-reactive protein (CRP) and creatine kinase (CK) levels on postoperative day 1 were also investigated.

Data were analyzed using the Wilcoxon signed-rank test for the assessment of postoperative improvement in each group and the Mann-Whitney $U$-test for the comparison between the groups. Data analyses were conducted using the SPSS ver. 21 (SPSS Japan Inc., IBM, Tokyo, Japan). Statistical significance was set at $p<0.05$.

\section{Results}

The patient data are summarized in Table 1. The mean follow-up period was $41.8 \pm 24.7$ months (range, $12-75$ months) in the LT retractors group and 33.0 19.3 months (range, 10-60 months) in the TL retractors group. The average operating time in the TL group ( $60.5 \pm 22.5$ minutes; range, 35-105 minutes) was significantly shorter than that in the LT group (86.2 \pm 30.0 minutes; range, $51-144$ minutes; $p=0.011$ ). No significant difference between the groups in terms of the average intraoperative blood loss (LT: $42.0 \pm 45.4 \mathrm{~g}$, range, $10-120 \mathrm{~g}$; TL: $43.5 \pm 43.8 \mathrm{~g}$, range, 10-100 g; $p=0.796$ ) occurred.

In the LT group, the mean preoperative and final follow-up NDI scores were 28.0 \pm 9.4 (range, 11-44) and $12.0 \pm 7.8$ (range, $0-23$ ), respectively, thus showing a sig-

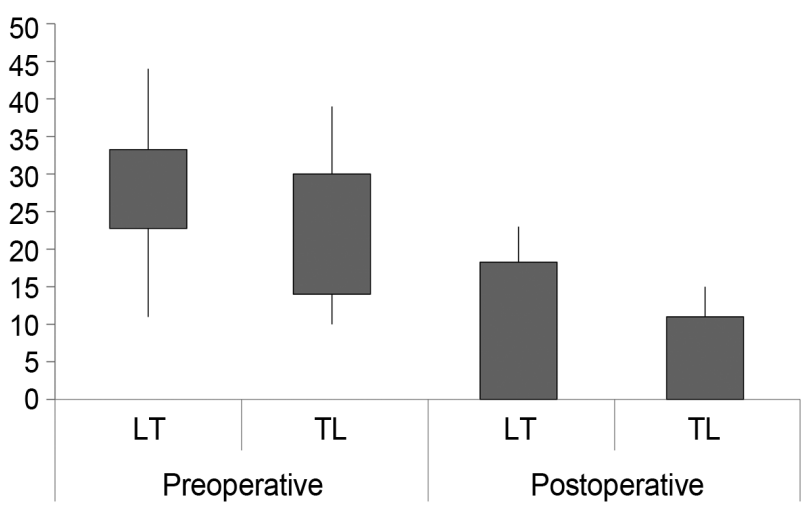

Fig. 2. Preoperative and postoperative neck disability index (NDI) scores. In the large tubular (LT) retractor group, the mean NDI score at the final follow-up was significantly improved from 28.0 to 12.0 $(p<0.001)$. In the TrimLine (TL) retractor group, the mean preoperative and final follow-up NDI scores were 21.9 and 6.0, respectively. The mean score improved significantly $(p=0.008)$.

nificant improvement $(p<0.001)$. Such an improvement was also observed in the TL group $(21.9 \pm 10.3$; range, $10-39$ vs. $6.0 \pm 5.9$; range, $0-15 ; p=0.008$ ) (Fig. 2 ). The recovery rates of NDI were $52.3 \% \pm 36.5 \%$ in the LT group and $68.3 \% \pm 31.0 \%$ in the TL group with no significant difference between the two groups ( $p=0.268$ ).

In the LT group, the VAS score for neck pain improved significantly from $8.0 \pm 1.5$ (range, 6-10) preoperatively to $2.2 \pm 2.2$ (range, $0-5$ ) at the final follow-up ( $p=0.003$ ). A significant improvement from $2.5 \pm 2.5$ (range, $0-6$ ) preoperatively to $1.0 \pm 2.5$ (range, $0-8$ ) at the final followup $(p=0.027)$ was also observed in the TL group. The recovery rates of the neck pain VAS score showed no significant difference $(70.0 \% \pm 31.9 \%$ in the LT group and $87.0 \% \pm 32.0 \%$ in the TL group, $p=0.125$ ).

In the LT group, the VAS score for arm pain improved significantly from 7.0 \pm 2.9 (range, $0-10$ ) preoperatively 
Table 2. Comparison of clinical results

\begin{tabular}{lccc}
\multicolumn{1}{c}{ Variable } & LT retractor group $(\mathrm{n}=10)$ & TL retractor group $(\mathrm{n}=10)$ & $p$-value ${ }^{\text {a) }}$ \\
\hline CRP on postoperative day 1 (mg/dL) & $0.46 \pm 0.61$ & $0.65 \pm 0.43$ & 0.064 \\
\hline CK on postoperative day 1 (mg/dL) & $250 \pm 191$ & $250 \pm 132$ & 0.360 \\
\hline Return to normal temperature (day) & $1.6 \pm 1.6$ & $1.0 \pm 0.8$ & 0.578 \\
\hline Length of hospital stay (day) & $11.4 \pm 3.3$ & $12.7 \pm 3.1$ & 0.269 \\
Recovery rate of VAS score for neck pain (\%) & $70.0 \pm 31.9$ & $87.0 \pm 32.0$ & 0.125 \\
Recovery rate of VAS score for arm pain (\%) & $76.9 \pm 34.4$ & $82.0 \pm 41.9$ & 0.488 \\
\hline
\end{tabular}

Values are presented as mean \pm standard deviation.

LT, large tubular; TL, TrimLine; CRP, C-reactive protein; CK, creatine kinase; VAS, visual analog scale.

a) Determined by the Mann-Whitney U-test.

to $1.4 \pm 1.9$ (range, $0-5)$ at the final follow-up ( $p=0.007$ ). In the TL group, this score also showed a significant improvement from $6.3 \pm 2.1$ (range, $4-10$ ) preoperatively to $0.8 \pm 1.7$ (range, $0-5)$ at the final follow-up $(p=0.004)$. No significance difference in the recovery rates of the arm pain VAS score was observed $(76.9 \% \pm 34.4 \%$ in the LT group and $82.0 \% \pm 41.9 \%$ in the TL group, $p=0.488$ ).

The mean time to return to normal body temperature $\left(<37^{\circ} \mathrm{C}\right)$ after the surgery was $1.6 \pm 1.6$ days in the LT group and $1.0 \pm 0.8$ days in the TL group with no significant difference between the two groups ( $p=0.578)$. The average lengths of the hospital stay were also similar $(11.4 \pm 3.3$ days in the LT group and 12.7 \pm 3.1 days in the TL group, $p=0.269$ ). The mean CRP and CK levels on postoperative day 1 were $0.46 \pm 0.61 \mathrm{mg} / \mathrm{dL}$ and $250 \pm 191 \mathrm{mg} / \mathrm{dL}$ in the LT group; $0.65 \pm 0.43 \mathrm{mg} / \mathrm{dL}$ and $250 \pm 132 \mathrm{mg} / \mathrm{dL}$ in the TL group, respectively. No significant differences between the two groups ( $p=0.064$ and $p=0.360$, respectively) occurred. The clinical results are compared in Table 2.

\section{Discussion}

Although the anterior operative approach is widely utilized for the treatment of cervical radiculopathy, the advantages of posterior cervical foraminotomy in selected cases of nerve root decompression in the posterolateral disc and for the treatment of foraminal stenosis have been well documented.

This approach avoids many of the hazards associated with anterior exposure including injury to the recurrent laryngeal nerve, trachea, esophagus, carotid sheath, and thoracic duct. Moreover, it prevents adjacent segment syndrome caused by unnecessary fusion $[8,9]$ and may provide better exposure for decompression of the exiting root and for removal of the lateral osteophytes and discs $[10,11]$. Nonetheless, operative trauma to the cervical paraspinal muscles associated with the posterior approach is a major cause of postoperative complications that may include persistent neck and shoulder pain and sometimes spinal instability [3-5]. MOF with LT retractors is a minimally invasive and effective surgical operation for cervical radiculopathy that reduces damage to the cervical paraspinal muscles [6].

Muscle injury during surgical procedures can cause an increase in CK levels [12-14] with some reports indicating increased CK activity after spinal surgery [15-17]. In our study, the mean CK level on postoperative day 1 was low, and no statistically significant differences were observed between the LT and TL groups ( $p=0.360)$. Furthermore, the mean CRP level was also low in both groups. These findings suggest that MOF is less invasive than the conventional surgical approaches.

The time required for setting a LT retractor was longer than that for a TL retractor. However, this difference in time can be compensated for if the LT retractor was inserted after making a $5 \mathrm{~cm}$ skin incision and detaching the paravertebral muscles from the spinous processes and lamina by electrocautery.

The NDI scores improved significantly at final followup in both the LT and TL groups $(p<0.001$ and 0.008 , respectively). Importantly, no significant difference in the recovery rates of NDI was found between the two groups $(p=0.268)$. This finding suggests that MOF using the TL retractor was comparable to MOF performed with a LT retractor in terms of clinical results. The VAS scores for neck pain improved significantly at final follow-up in both the LT and TL groups ( $p=0.003$ and 0.027 , respectively) although the preoperative VAS score in TL group 
was incidentally lower, which is one of limitations.

This study was limited by its small sample size, short follow-up period, not comparing conventional surgical approach, and retrospective design. Importantly, although the clinical results and surgical invasiveness were similar when MOF was performed using LT or TL retractors, the operating time was significantly lower in the TL group. Large TLs were mainly used in the beginning of the study whereas TL retractors were increasingly employed in the recent years. Therefore, we believe that the possible bias coming from the learning curve cannot be ignored. Overall, MOF with TL retractors, which can be performed through a $5 \mathrm{~cm}$ skin incision, retained the low invasiveness of conventional MOF. Furthermore, this procedure required less experience than either endoscopic surgery or MOF involving LT retractors because it was performed under direct vision. Therefore, it can be highly effective in patients with cervical radiculopathy secondary to posterolateral disc herniation or foraminal spondylotic stenosis.

\section{Conclusions}

Mini open foraminotomy, involving TL retractors, is an easy and safe procedure. Furthermore, this procedure was less invasive and more effective for patients with cervical radiculopathy secondary to posterolateral disc herniation or foraminal spondylotic stenosis.

\section{Conflict of Interest}

No potential conflict of interest relevant to this article was reported.

\section{References}

1. Frykholm R. Cervical nerve root compression resulting from disc degeneration and root sleeve fibrosis. Acta Chir Scand Suppl 1951;160:1-149.

2. Scoville WB. Cervical spondylosis treated by bilateral facetectomy and laminectomy. J Neurosurg 1961;18:423-8.

3. Hosono N, Yonenobu K, Ono K. Neck and shoulder pain after laminoplasty: a noticeable complication. Spine 1996;21:1969-73.

4. Kawaguchi Y, Matsui H, Ishihara H, Gejo R, Yoshino O. Axial symptoms after en bloc cervical laminoplas- ty. J Spinal Disord 1999;12:392-5.

5. Zdeblick TA, Zou D, Warden KE, McCabe R, Kunz D, Vanderby R. Cervical stability after foraminotomy: a biomechanical in vitro analysis. J Bone Joint Surg Am 1992;74:22-7.

6. Takahashi J, Aoki K, Ogihara N, Hirabayashi H, Hashidate $\mathrm{H}$, Kato $\mathrm{H}$. Mini open foraminotomy (MOF) for cervical radiculopathy: technical note. Open Spine J 2009;1:1-4.

7. Hirabayashi K, Watanabe K, Wakano K, Suzuki N, Satomi K, Ishii Y. Expansive open-door laminoplasty for cervical spinal stenotic myelopathy. Spine (Phila Pa 1976) 1983;8:693-9.

8. Hunter L, Braunstein E, Bailey R. Radiographic changes following anterior cervical fusion. Spine (Phila Pa 1976) 1980;5:399-401.

9. Olsewski JM, Garvey TA, Schendel MJ. Biomechanical analysis of facet and graft loading in a SmithRobinson type cervical spine model. Spine (Phila Pa 1976) 1994;19:2540-4.

10. Reinhardt HF, Ruetschle M, Stricker E, Gratzl O. Results of the surgical treatment of cervical disk hernia and myelopathy with dorsal approach. Fortschr Med 1983;101:979-85.

11. Roh SW, Kim DH, Cardoso AC, Fessler RG. Endoscopic foraminotomy using MED system in cadaveric specimens. Spine (Phila Pa 1976) 2000;25:260-4.

12. Nevins MA, Saran M, Bright M, Lyon LJ. Pitfalls in interpreting serum creatine phosphokinase activity. JAMA 1973;224:1382-7.

13. Penneys R, Wilkinson JH. Elevation of serum creatine kinase following amputation of the leg. Surgery 1970;67:302-5.

14. Savignano T, Hanok A, Kuo J. Creatine phosphokinase activity: a study of normal and abnormal levels. Am J Clin Pathol 1969;51:76-85.

15. Lenke LG, Bridwell KH, Jaffe AS. Increase in creatine kinase $\mathrm{MB}$ isoenzyme levels after spinal surgery. J Spinal Disord 1994;7:70-6.

16. Healey JH, Kagen LJ, Velis KP, Levine DB. Creatine kinase $\mathrm{MB}$ in skeletal muscle and serum of spine-fusion patients. Clin Orthop Relat Res 1985;(195):282-8.

17. Wukich DK, Van Dam BE, Graeber GM, Martyak T. Serum creatine kinase and lactate dehydrogenase changes after anterior approaches to the thoracic and lumbar spine. Spine (Phila Pa 1976) 1990;15:187-90. 